CORRIGENDUM

doi:10.1038/nature10675

\title{
A draft genome of Yersinia pestis from victims of the Black Death
}

Kirsten I. Bos, Verena J. Schuenemann, G. Brian Golding, Hernán A. Burbano, Nicholas Waglechner, Brian K. Coombes, Joseph B. McPhee, Sharon N.DeWitte, Matthias Meyer, Sarah Schmedes, JamesWood, David J. D. Earn, D. Ann Herring, Peter Bauer, Hendrik N. Poinar \& Johannes Krause

Nature 478, 506-510 (2011)

In this Letter, the GenBank accession number was wrongly printed as SRA045745.1. It should be SRA045745. Supplementary Tables 3a and 4 have also been corrected. 